21st Particles and Nuclei International Conference (PANIC 2017)

International Journal of Modern Physics: Conference Series

Vol. 46 (2018) 1860031 (6 pages)

(C) The Author(s)

DOI: $10.1142 / \mathrm{S} 2010194518600315$

\title{
Heavy Flavour Spectroscopy at LHCb
}

\author{
Ao $\mathrm{Xu}$ (on behalf of $\mathrm{LHCb}$ collaboration) \\ Department of Engineering Physics, Tsinghua University \\ 30 Shuangqing Rd, 100084 Beijing, China \\ Center for High Energy Physics, Tsinghua University \\ 30 Shuangqing Rd, 100084 Beijing, China \\ xua17@mails.tsinghua.edu.cn
}

Published 3 May 2018

\begin{abstract}
Recent results on heavy flavour spectroscopy with data collected by the LHCb detector are highlighted, including studies of exotic hadrons, the observation of five $\Omega_{c}^{0}$ resonances, and the observation of the $\Xi_{c c}^{++}$baryon.
\end{abstract}

Keywords: Heavy flavour physics; Spectroscopy; Large Hadron Collider.

\section{Introduction}

Study of hadron spectroscopy can provide invaluable information for quantum chromodynamics, which describes strong interactions between quarks and gluons. This article reports recent results on heavy flavour spectroscopy from the LHCb experiment. The $\mathrm{LHCb}$ detector is a single-arm forward spectrometer designed for studies of particles containing $b$ or $c$ quarks, described in detail in Refs. 1, 2 .

In LHC Run 1, the detector collected $p p$ collision data corresponding to an integrated luminosity of about $1(2) \mathrm{fb}^{-1}$ at $\sqrt{s}=7$ (8) TeV in 2011 (2012). During LHC Run 2, a data sample corresponding to an integrated luminosity of about 0.3 (1.7) $\mathrm{fb}^{-1}$ at $\sqrt{s}=13 \mathrm{TeV}$ are collected in 2015 (2016).

\section{Heavy exotic hadron spectroscopy}

\subsection{Observation of the decays $\Lambda_{b}^{0} \rightarrow \chi_{c J} p K^{-}$}

LHCb observed two pentaquark candidates $P_{c}(4380)^{+}$and $P_{c}(4450)^{+}$in the amplitude analysis of $\Lambda_{b}^{0} \rightarrow J / \psi p K^{-}$decays in $2015^{3}$, which were confirmed by a model-independent analysis ${ }^{4}$. (Inclusion of charge-conjugate processes is implied

This is an Open Access article published by World Scientific Publishing Company. It is distributed under the terms of the Creative Commons Attribution 4.0 (CC-BY) License. Further distribution of this work is permitted, provided the original work is properly cited. 
A. $X u$
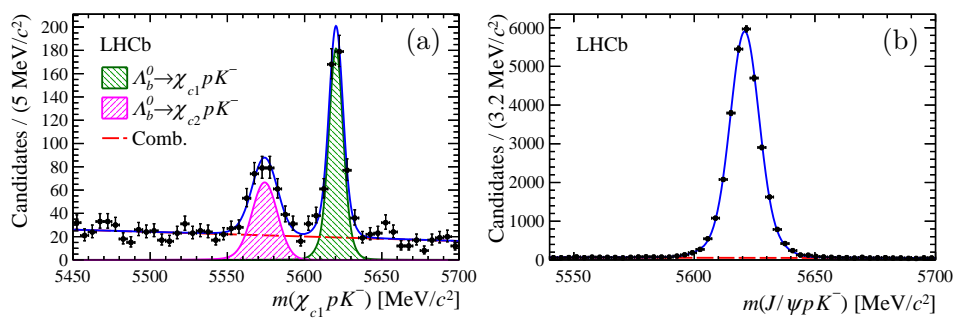

Fig. 1. Fits to the (a) $\Lambda_{b}^{0} \rightarrow \chi_{c 1} p K^{-}$and (b) $\Lambda_{b}^{0} \rightarrow J / \psi p K^{-}$invariant mass distributions.

throughout.) The $P_{c}(4450)^{+}$mass is close to the $\chi_{c 1} p$ threshold, which leaves the possibility of describing the state as kinematic rescattering effects. This could be tested by the analysis of the decay $\Lambda_{b}^{0} \rightarrow \chi_{c 1} p K^{-}$.

As an initial stage in the investigation of this hypothesis, the observation of $\Lambda_{b}^{0} \rightarrow \chi_{c J} p K^{-}$decays is achieved using the Run 1 data sample, where $J=1,2$, and the branching fractions relative to that of $\Lambda_{b}^{0} \rightarrow J / \psi p K^{-}$decays are measured ${ }^{5}$. In the decay chains $\Lambda_{b}^{0} \rightarrow \chi_{c J} p K^{-}$and $\Lambda_{b}^{0} \rightarrow J / \psi p K^{-}$, the intermediate resonances $\chi_{c J}$ and $J / \psi$ are reconstructed with the decays $\chi_{c J} \rightarrow J / \psi \gamma$ and $J / \psi \rightarrow \mu^{+} \mu^{-}$. A kinematic fit is applied to the $\Lambda_{b}^{0}$ candiates with $\chi_{c 1}$ and $J / \psi$ masses constraint to their known values. As a result, Separate peaks are produced in the $m\left(\chi_{c 1} p K^{-}\right)$distribution. The signal yields of the modes are determined by the fits to the $m\left(\chi_{c 1} p K^{-}\right)$and $m\left(J / \psi p K^{-}\right)$distributions shown in Fig. 1. The measured yields of $\Lambda_{b}^{0}$ are $453 \pm 25,285 \pm 23$, and $29815 \pm 178$ for the $\chi_{c 1}, \chi_{c 2}$, and $J / \psi$ channels, respectively. The ratios of branching fractions are found to be

$$
\begin{aligned}
& \frac{\mathcal{B}\left(\Lambda_{b}^{0} \rightarrow \chi_{c 1} p K^{-}\right)}{\mathcal{B}\left(\Lambda_{b}^{0} \rightarrow J / \psi p K^{-}\right)}=0.242 \pm 0.014 \pm 0.013 \pm 0.009, \\
& \frac{\mathcal{B}\left(\Lambda_{b}^{0} \rightarrow \chi_{c 2} p K^{-}\right)}{\mathcal{B}\left(\Lambda_{b}^{0} \rightarrow J / \psi p K^{-}\right)}=0.248 \pm 0.020 \pm 0.014 \pm 0.009, \text { and } \\
& \frac{\mathcal{B}\left(\Lambda_{b}^{0} \rightarrow \chi_{c 2} p K^{-}\right)}{\mathcal{B}\left(\Lambda_{b}^{0} \rightarrow \chi_{c 1} p K^{-}\right)}=1.02 \pm 0.10 \pm 0.02 \pm 0.05,
\end{aligned}
$$

where the uncertainties are due to statistical, systematic, and the uncertainty on the branching fractions of the $\chi_{c J} \rightarrow J / \psi \gamma$ decays, respectively. Unlike in $B \rightarrow \chi_{c J} K$ decays ${ }^{6,7}$, no suppression of the $\chi_{c 2}$ mode relative to $\chi_{c 1}$ mode is observed. The amplitude analysis of the $\Lambda_{b}^{0} \rightarrow \chi_{c 1} p K^{-}$decay is under way.

\subsection{Observation of $J / \psi \phi$ structures}

Both positive and negative results of searches for the $X(4140)$ structure in the decay $B^{+} \rightarrow J / \psi \phi K^{+}$have been reported by various experiments ${ }^{8,9,10,11,12}$. An amplitude analysis of this decay could help to achieve a better understanding by considering the reflections of $K^{*}$ states in the $J / \psi \phi$ invariant mass distribution. This 

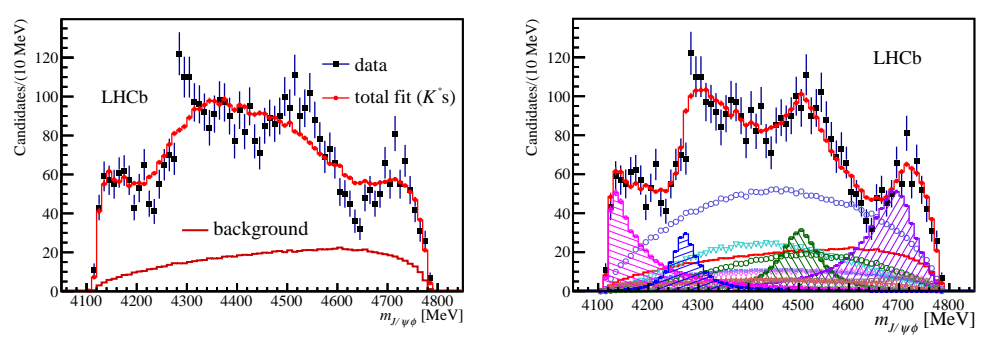

Fig. 2. Distributions of $m_{J / \psi \phi}$ for the fit results with (left) model containing only $K^{*+}$ contributions and (right) model containing eight $K^{*+}$ and five $X$ contributions.

Table 1. Results for significances, masses, widths, and quantum numbers of the exotic states in the best-fit model.

\begin{tabular}{lcccc}
\hline Resonance & Significance & Mass $(\mathrm{MeV})$ & $\Gamma(\mathrm{MeV})$ & $J^{P C}$ (Significance) \\
\hline$X(4140)$ & $8.4 \sigma$ & $4146.5 \pm 4.5_{-2.8}^{+4.6}$ & $83 \pm 21_{-14}^{+21}$ & $1^{++}(5.7 \sigma)$ \\
$X(4274)$ & $6.0 \sigma$ & $4273.3 \pm 8.3_{-3.6}^{+17.2}$ & $56.2 \pm 10.9_{-11.1}^{+8.4}$ & $1^{++}(5.8 \sigma)$ \\
$X(4500)$ & $6.1 \sigma$ & $4506 \pm 11_{15}^{+12}$ & $92 \pm 21_{20}^{+21}$ & $0^{++}(4.0 \sigma)$ \\
$X(4700)$ & $5.6 \sigma$ & $4704 \pm 10_{-24}^{+14}$ & $120 \pm 31_{-33}^{+42}$ & $0^{++}(4.5 \sigma)$ \\
\hline
\end{tabular}

is performed by $\mathrm{LHCb}$ using the full Run 1 data ${ }^{13,14}$. The yield of $B^{+} \rightarrow J / \psi \phi K^{+}$ events after selection is $4289 \pm 151$, with a background fraction of $(23 \pm 6) \%$ in the $B^{+}$mass region used in the amplitude analysis.

The left plot of Fig. 2 shows that the amplitude model with kaon excitations alone failed to describe the data in the $m_{J / \psi \phi}$ distribution. The best-fit resonance model contains seven $K^{*+}$ states, four $X$ states, and $\phi K^{+}$and $J / \psi \phi$ nonresonant components, with 98 free parameters in the fit. The fit projection on the $m_{J / \psi \phi}$ plane is shown in the right plot of Fig. 2. Four exotic states contribute in the model, with parameters summarized in Table 1.

\subsection{Search for structure in the $B_{s}^{0} \pi^{ \pm}$mass spectrum}

The D0 experiment claimed evidence of a narrow structure in the $B_{s}^{0} \pi^{ \pm}$mass spectrum, referred to as $X(5568)^{15}$. If confirmed, $X(5568)$ would provide important information of the bounding mechanism of the multiquark structure, since its mass is dominated by one heavy quark instead of the heavy quark-antiquark pair of previously observed exotic states.

The search has been conducted using Run 1 data ${ }^{16}$. The $B_{s}^{0}$ samples are reconstructed through decays to $D_{s}^{-} \pi^{+}$with $D_{s}^{-} \rightarrow K^{+} K^{-} \pi^{-}$, and decays to $J / \psi \phi$ with $J / \psi \rightarrow \mu^{+} \mu^{-}$and $\phi \rightarrow K^{+} K^{-}$. After the event selection, a $B_{s}^{0}$ sample of about $10^{5}$ mesons is acquired, which is 20 times larger than that used by the D0 experiment. A fit to the $B_{s}^{0} \pi^{ \pm}$mass spectrum has been performed to obtain quantitative results. No significant signal is observed. The upper limits at 90(95)\% C.L. of the ratio of cross-section, $\rho_{X}^{\mathrm{LHCb}} \equiv \frac{\sigma(p p \rightarrow X+\text { anything }) \times \mathcal{B}\left(X \rightarrow B_{s}^{0} \pi^{ \pm}\right)}{\sigma\left(p p \rightarrow B_{s}^{0}+\text { anything }\right)}$, are evaluated to be 


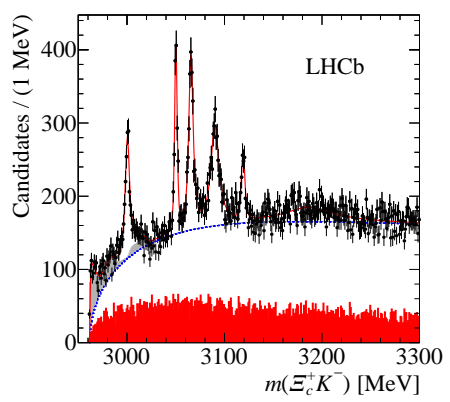

Fig. 3. The distribution of $m\left(\Xi_{c}^{+} K^{-}\right)$of the reconstructed candidates passing the likelihood ratio selection.

$\rho_{X}^{\mathrm{LHCb}}\left[p_{T}\left(B_{s}^{0}\right)>5 \mathrm{GeV}\right]<0.011(0.012), \rho_{X}^{\mathrm{LHCb}}\left[p_{T}\left(B_{s}^{0}\right)>10 \mathrm{GeV}\right]<0.021(0.024)$, and $\rho_{X}^{\mathrm{LHCb}}\left[p_{T}\left(B_{s}^{0}\right)>15 \mathrm{GeV}\right]<0.018(0.020)$.

\section{Heavy conventional hadron spectroscopy}

\subsection{Observation of five excited $\Omega_{c}^{0}$ states}

Compared to other charmed baryons, our knowledge of $\Omega_{c}^{0}$ spectra is very limited. Only the ground states of $J^{P}=1 / 2^{+} \Omega_{c}^{0}$ and $J^{P}=3 / 2^{+} \Omega_{c}(2770)^{0}$ are observed. A search for new $\Omega_{c}^{0}$ resonances decaying to $\Xi_{c}^{+} K^{-}$is performed with Run 1 and 2015 data ${ }^{17}$. The $\Xi_{c}^{+}$candidates are reconstructed with $p K^{-} \pi^{+}$final states.

The distribution of $m\left(\Xi_{c}^{+} K^{-}\right)$is shown in Fig. 3, where five narrow structures are observed. Further checks show that no combinatorial background, other resonances and mis-identification candidates contribute to theses narrow structures. The mass, width, and yield of each resonance are extracted through a binned $\chi^{2}$ fit to the $m\left(\Xi_{c}^{+} K^{-}\right)$spectrum in the range from the threshold to $3450 \mathrm{MeV}$. Resonances are described by spin-zero relativistic Breit-Wigner functions convolved with the experimental resolution. Feed-down contributions due to partial reconstruction are included with shape parameters fixed to simulation results. The fit results are shown in Table 2. Future investigations can determine the quantum numbers by studying possible three-body decays and reconstructing these states in decays of heavy baryons.

\subsection{Observation of the doubly charmed baryon $\Xi_{c c}^{++}$}

Doubly charmed baryons are predicted by the quark model, including one isospin doublet $\left(\Xi_{c c}^{++}(c c u)\right.$ and $\left.\Xi_{c c}^{+}(c c d)\right)$ and one isospin singlet $\left(\Omega_{c c}^{+}(c c s)\right)$. Experimentally, there is a long-standing puzzle in the $\Xi_{c c}$ system. An observation of $\Xi_{c c}^{+}$baryons was reported by the SELEX experiment in two decay modes, with a few unexpected features ${ }^{18,19}$. Searches at other experiments, however, reported negative results of the structure claimed by SELEX $20,21,22,23$.

The search for $\Xi_{c c}^{++} \rightarrow \Lambda_{c}^{+} K^{-} \pi^{+} \pi^{+}$decays is conducted with 2016 data $^{24}$. The $\Lambda_{c}^{+}$candidates are reconstructed through $p K^{-} \pi^{+}$final states. A significant 
Table 2. Results of the fit to $m\left(\Xi_{c}^{+} K^{-}\right)$for the mass, width, and yield for each resonance.

\begin{tabular}{lccc}
\hline Resonance & Mass $(\mathrm{MeV})$ & $\Gamma(\mathrm{MeV})$ & Yield \\
\hline$\Omega_{c}(3000)^{0}$ & $3000.4 \pm 0.2 \pm 0.1_{-0.5}^{+0.3}$ & $4.5 \pm 0.6 \pm 0.3$ & $1300 \pm 100 \pm 80$ \\
$\Omega_{c}(3050)^{0}$ & $3050.2 \pm 0.1 \pm 0.1_{-0.5}^{+0.3}$ & $0.8 \pm 0.2 \pm 0.1$ & $970 \pm 60 \pm 20$ \\
$\Omega_{c}(3066)^{0}$ & $3065.6 \pm 0.1 \pm 0.3_{-0.5}^{+0.3}$ & $3.5 \pm 0.4 \pm 0.2$ & $1740 \pm 100 \pm 50$ \\
$\Omega_{c}(3090)^{0}$ & $3090.2 \pm 0.3 \pm 0.5_{-0.5}^{+0.3}$ & $8.7 \pm 1.0 \pm 0.8$ & $2000 \pm 140 \pm 130$ \\
$\Omega_{c}(3119)^{0}$ & $3119.1 \pm 0.3 \pm 0.9_{-0.5}^{+0.3}$ & $1.1 \pm 0.8 \pm 0.4$ & $480 \pm 70 \pm 30$ \\
\hline
\end{tabular}
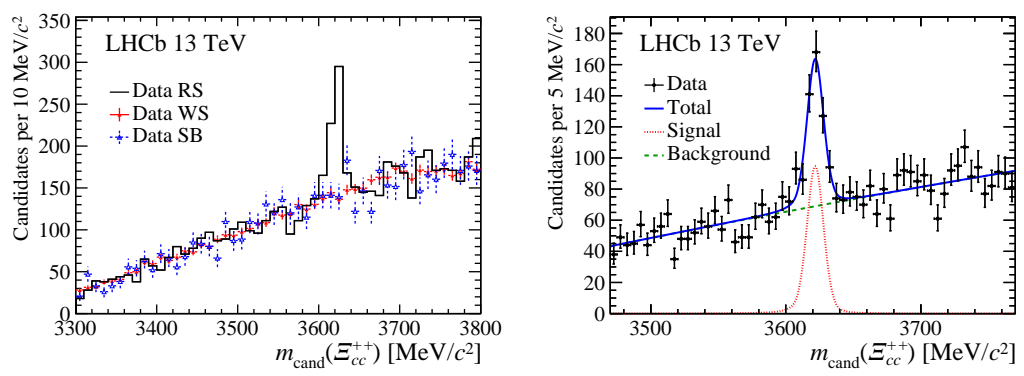

Fig. 4. Distributions of (left) the invariant mass of $\Lambda_{c}^{+} K^{-} \pi^{+} \pi^{+}$for right-sign (RS) signal sample, $\Lambda_{c}^{+} K^{-} \pi^{+} \pi^{-}$for wrong-sign (WS) control sample, and $\left[p K^{-} \pi^{+}\right] K^{-} \pi^{+} \pi^{+}$with $\left[p K^{-} \pi^{+}\right]$combinations in the $\Lambda_{c}^{+}$mass sidebands for side-band (SB) control sample and (right) the fit result in the mass window.

structure is observed at around $3620 \mathrm{MeV}$ in the $\Xi_{c c}^{++}$mass spectrum, while no structure is found in the control samples, as is shown in the left plot of Fig. 4. The same structure is also observed in the data sample collected in 2012. To determine the yield and significance of the structure, as well as the peak mass, an unbinned extended maximum likelihood fit is performed in the mass window $3620 \pm 150 \mathrm{MeV}$, as is shown in the right plot of Fig. 4. The signal significance is above $12 \sigma$ with a yield of $313 \pm 33$. The measured mass is $3621.40 \pm 0.72 \pm 0.27 \pm 0.14 \mathrm{MeV}$, where the uncertainties are statistical, systematic, and due to the uncertainty of the $\Lambda_{c}^{+}$ mass, respectively.

Possible mis-identification contributions are found to be negligible. In addition, when requiring the decay time larger than 5 times the resolution, the significance of signal is still above $12 \sigma$, which confirms the inconsistency with a strong decay. The mass of the observed $\Xi_{c c}^{++}$is consistent with most theoretical predictions, while inconsistent (103 $\pm 2 \mathrm{MeV}$ larger) with being an isospin partner with the structure reported by the SELEX experiment.

\section{Conclusion and prospects}

Rich results of heavy flavour spectroscopy have been produced by LHCb, and recent results are highlighted. More exciting results are expected with enlarged data sample. 


\section{References}

1. A. A. Alves Jr. et al. The LHCb detector at the LHC. JINST, 3:S08005, 2008.

2. R. Aaij et al. LHCb detector performance. Int. J. Mod. Phys., A30:1530022, 2015.

3. R. Aaij et al. Observation of $J / \psi p$ resonances consistent with pentaquark states in $\Lambda_{b}^{0} \rightarrow J / \psi p K^{-}$decays. Phys. Rev. Lett., 115:072001, 2015.

4. R. Aaij et al. Model-independent evidence for $J / \psi p$ contributions to $\Lambda_{b}^{0} \rightarrow J / \psi p K^{-}$ decays. Phys. Rev. Lett., 117:082002, 2016.

5. R. Aaij et al. Observation of the decays $\Lambda_{b}^{0} \rightarrow \chi_{c 1} p K^{-}$and $\Lambda_{b}^{0} \rightarrow \chi_{c 2} p K^{-}$. Phys. Rev. Lett., 119:062001, 2017.

6. R. Mizuk et al. Observation of two resonancelike structures in the $\pi^{+} \chi_{c 1}$ mass distribution in exclusive $\bar{B}^{0} \rightarrow K^{-} \pi^{+} \chi_{c 1}$ decays. Phys. Rev., D78:072004, 2008.

7. R. Aaij et al. Observation of $B_{s}^{0} \rightarrow \chi_{c 1} \phi$ decay and study of $B^{0} \rightarrow \chi_{c 1,2} K^{* 0}$ decays. Nucl. Phys., B874:663, 2013.

8. T. Aaltonen et al. Evidence for a narrow near-threshold structure in the $J / \psi \phi$ mass spectrum in $B^{+} \rightarrow J / \psi \phi K^{+}$decays. Phys. Rev. Lett., 102:242002, Jun 2009.

9. Serguei Chatrchyan et al. Observation of a peaking structure in the $J / \psi \phi$ mass spectrum from $B^{ \pm} \rightarrow J / \psi \phi K^{ \pm}$decays. Phys. Lett., B734:261-281, 2014.

10. V. Abazov et al. Search for the $X(4140)$ state in $B^{+} \rightarrow J / \psi \phi K^{+}$decays with the D0 detector. Phys. Rev. D, 89:012004, Jan 2014.

11. R. Aaij et al. Search for the $X(4140)$ state in $B^{+} \rightarrow J / \psi \phi K^{+}$decays. Phys. Rev., D85:091103(R), 2012.

12. J. P. Lees et al. Study of $B^{ \pm, 0} \rightarrow J / \psi K^{+} K^{-} K^{ \pm, 0}$ and search for $B^{0} \rightarrow J / \psi \phi$ at BaBar. Phys. Rev. D, 91:012003, Jan 2015.

13. R. Aaij et al. Observation of exotic $J / \psi \phi$ structures from amplitude analysis of $B^{+} \rightarrow$ $J / \psi \phi K^{+}$decays. Phys. Rev. Lett., 118:022003, 2016.

14. R. Aaij et al. Amplitude analysis of $B^{+} \rightarrow J / \psi \phi K^{+}$decays. Phys. Rev., D95:012002, 2016.

15. V. M. Abazov et al. Evidence for a $B_{s}^{0} \pi^{ \pm}$state. Phys. Rev. Lett., 117:022003, Jul 2016.

16. R. Aaij et al. Search for structure in the $B_{s}^{0} \pi^{ \pm}$invariant mass spectrum. Phys. Rev. Lett., 117:152003, 2016.

17. R. Aaij et al. Observation of five new narrow $\Omega_{c}^{0}$ states decaying to $\Xi_{c}^{+} K^{-}$. Phys. Rev. Lett., 118:182001, 2017.

18. M. Mattson et al. First observation of the doubly charmed baryon $\Xi_{c c}^{+}$. Phys. Rev. Lett., 89:112001, Aug 2002.

19. A. Ocherashvili et al. Confirmation of the doubly charmed baryon $\Xi_{c c}^{+}(3520)$ via its decay to $p D^{+} K^{-}$. Physics Letters B, 628(1):18 - 24, 2005.

20. Sergio P. Ratti et al. New results on c-baryons and a search for cc-baryons in FOCUS. Nuclear Physics B - Proceedings Supplements, 115(Supplement C):33 - 36, 2003. Hyperons, Charm and Beauty Hadrons.

21. B. Aubert et al. Search for doubly charmed baryons $\Xi_{c c}^{+}$and $\Xi_{c c}^{++}$in BABAR. Phys. Rev. D, 74:011103, Jul 2006.

22. R. Chistov et al. Observation of new states decaying into $\Lambda_{c}^{+} K^{-} \pi^{+}$and $\Lambda_{c}^{+} K_{S}^{0} \pi^{-}$. Phys. Rev. Lett., 97:162001, Oct 2006.

23. R. Aaij et al. Search for the doubly charmed baryon $\Xi_{c c}^{+}$. JHEP, 12:090, 2013.

24. R. Aaij et al. Observation of the doubly charmed baryon $\Xi_{c c}^{++}$. Phys. Rev. Lett., 119:112001, 2017. 